\title{
Microscopic dynamics in liquid binary alloys: orbital-free ab-initio molecular dynamics studies
}

\author{
D.J.González, L.E.González \\ Departamento de Física Teórica, Universidad de Valladolid, 47011 Valladolid, Spain
}

Received October 30, 2007

\begin{abstract}
We report an ab-initio molecular dynamics study on the collective excitations in several $s-p$ bonded liquid binary alloys. Results are reported for the $\mathrm{Li}-\mathrm{Na}, \mathrm{Li}-\mathrm{Mg}, \mathrm{K}-\mathrm{Cs}$ and $\mathrm{Li}-\mathrm{Ba}$ liquid alloys at different concentrations, which display mass ratios ranging from $\approx 3$ for $\mathrm{Li}-\mathrm{Na}$ to $\approx 20$ for $\mathrm{Li}-\mathrm{Ba}$, and varying ordering tendencies, ranging from strong homocoordinating in $\mathrm{Li}-\mathrm{Na}$ to mildly heterocoordinating for one concentration of $\mathrm{Li}-\mathrm{Ba}$. The study has been carried out using the orbital free ab-initio molecular dynamics method, combined with local ionic pseudopotentials constructed within the same framework. We analyze the dependence of the collective modes on the concentration and the mass ratio of the alloy, finding a common behaviour for all systems notwithstanding the different ordering tendencies.
\end{abstract}

Key words: ab initio simulations, binary alloys, collective dynamics, longitudinal collective excitations, transverse collective excitations

PACS: $61.25 . \mathrm{Mv}, 61.55 . \mathrm{Hg}, 61.20 . \mathrm{Ja}$

\section{Introduction}

Molecular dynamics (MD) has become a basic tool in the study of liquid systems. Classical molecular dynamics (CMD) methods require interatomic potentials to calculate the forces on the atoms/ions whereas the ab-initio molecular dynamics (AIMD) methods compute those forces from electronic structure calculations which are performed as the MD trajectory is generated.

Density Functional Theory (DFT) $[1,2]$ underlies most AIMD methods. Starting with a collection of atoms/ions at given nuclear positions, DFT makes it possible to calculate the ground state electronic energy and, via the Hellmann-Feynman theorem, the forces acting on the atoms/ions. Within DFT, most methods use the Kohn-Sham (KS) orbital representation of DFT (KS-AIMD methods) [2] which is computationally demanding, whereby allowing the study of small sample sizes (one or two hundreds of particles) during short simulation times (few tens of ps). However, these constraints may be somewhat overcome by the so-called orbital-free ab initio molecular dynamics (OF-AIMD) method, which uses the Hohenberg-Kohn (HK) representation of the DFT [1], eliminates the electronic orbitals and permits to perform simulations with large samples (up to a few thousands of particles) and for long times (hundreds of ps).

Research into the dynamical properties of liquid metals has already produced a considerable amount of both experimental and theoretical work [3]. Inelastic neutron scattering (INS) has been the usual experimental technique for studying the dynamics of liquids at the kinetic region. Recently, it has been supplemented by the advent of high resolution inelastic X-ray scattering (IXS) techniques, which skip some restrictions posed by the INS techniques, such as the kinematic limitations as well as the presence of both coherent and incoherent contributions to the inelastic scattering cross section. On the other hand, the development of microscopic theories such as the memory function formalism or the kinetic theory, along with the realization that the decay of several time-dependent properties can be linked to the interplay of two different dynamical processes, has created a theoretical framework whose application to simple liquids has led to good qualitative results for several dynamical magnitudes [3-5].

Comparatively less effort has been devoted to the dynamical properties in liquid binary alloys. Most developments have occurred for the last twenty five years, starting with the pionering CMD 
studies on liquid $\mathrm{Na}-\mathrm{K}[6]$. It was followed by the $\mathrm{CMD}$ results for liquid $\mathrm{Li}_{4} \mathrm{~Pb}[7]$, where a new, high-frequency mode, allegedly supported by the light Li atoms only, (the so-called "fast sound") was found. This finding spurred a wealth of experimental [8-13], computer simulation [8,14-17] and theoretical [18-22] works focused on the investigation of the nature and properties of the collective excitations in liquid binary systems.

Evidence for the fast sound has already been obtained for systems such as $\mathrm{H}_{2}-\mathrm{Ar}, \mathrm{H}_{2}-\mathrm{Xe}$ and $\mathrm{He}-\mathrm{Xe}$ mixtures by light scattering [13], and $\mathrm{He}-\mathrm{Ne}, \mathrm{He}-\mathrm{Ar}$ and $\mathrm{Li}_{4} \mathrm{~Pb}$ by INS $[8,15,20]$. Excepting $\mathrm{Li}_{4} \mathrm{~Pb}$ which is a pseudoionic mixture, all the other systems are gas mixtures, where the mass ratio varies from around 5 for He-Ne to approximately 33 for He-Xe. A controversial point has been the way in which the two collective modes connect with the hydrodynamic one when $q_{\mathrm{h}}$ (which denotes the upper limit of the hydrodynamic limit) is approached from high $q$-values. Several theoretical and CMD results for $\mathrm{He}-\mathrm{Ar}$ and $\mathrm{He}-\mathrm{Ne}$ mixtures have suggested that either the fast and slow sound merge into the hydrodynamic sound $[8,14]$, or that the fast sound disappears at $q_{\mathrm{h}}$ and the slow sound merges into the hydrodynamic one with a predicted value $[19,20]$ of $q_{\mathrm{h}} \approx 0.07 \AA^{-1}$. A similar value for $q_{\mathrm{h}}$ has recently been obtained for liquid $\mathrm{Li}_{4} \mathrm{~Pb}$ by both INS and by CMD calculations $[11,15]$. Recent CMD calculations [16], which followed the INS measurements of Bafile et al. [12], for the $\mathrm{He}_{0.77} \mathrm{Ne}_{0.23}$ gas mixture at two densities, showed a clear crossover from hydrodynamics to fast and slow modes at $0.2 \leqslant q / \AA^{-1} \leqslant 0.5$; moreover, it was obtained that $q_{\mathrm{h}} \approx 0.2$, which is substantially greater than previous estimates.

On the theoretical side, kinetic theory calculations [18] for two-component fluids with large atomic mass difference, have confirmed the appearance of a collective mode at high frequencies and wave numbers $q \geqslant q_{\mathrm{h}}$. Moreover, this mode propagated with a phase velocity close to that of the pure light component, which was clearly greater than the hydrodynamic sound velocity, $c_{h}$, of the two-component system. Subsequently, calculations based on the revised Enskog theory (RET) for binary mixtures of hard spheres with a large mass difference [19] have predicted two propagating collective modes, in addition to hydrodynamic sound. One of these was identified with the fast sound while the other mode, which had a propagating phase velocity below $c_{h}$, was named "slow sound".

The transverse dynamics has been much less investigated, mainly because it is not visible in scattering experiments and only MD simulations can provide insight into the transverse excitations. The recent application of the generalized collective model (GCM) approach [21], which combines CMD simulations with the memory function formalism, to binary Lennard-Jones fluids and liquid alloys has unveiled the existence of transverse optic modes, which arise in connection with the concentration fluctuations.

This paper reports ab-initio studies on the longitudinal and transverse collective dynamics in several liquid binary alloys. The study has focused on $s$ - $p$ bonded liquid binary alloys for which the OF-AIMD method has already proved its capability to tackle a wide range of static and dynamic properties.

\section{Theory}

A simple liquid metallic alloy, $\mathrm{A}_{1-x} \mathrm{~B}_{x}$, can be regarded as an assembly of $N_{\mathrm{A}}$, A-type, and $N_{\mathrm{B}}$, B-type, bare ions with charges $Z_{v}^{\mathrm{A}}$ and $Z_{v}^{\mathrm{B}}$ respectively, interacting with $N_{e}=N_{\mathrm{A}} Z_{v}^{\mathrm{A}}+N_{\mathrm{B}} Z_{v}^{\mathrm{B}}$ valence electrons through electron-ion potentials $v_{\mathrm{A}}(r)$ and $v_{\mathrm{B}}(r)$. Therefore, the total potential energy of the system can be written, within the Born-Oppenheimer approximation, as the sum of the direct ion-ion coulombic interaction energy, and the ground state energy of the electronic system on the external potential created by the ions, $V_{\text {ext }}\left(\vec{r},\left\{\vec{R}_{l}\right\}\right)=\sum_{i=\mathrm{A}, \mathrm{B}} \sum_{l(i)} v_{i}\left(\left|\vec{r}-\vec{R}_{l}\right|\right)$,

$$
E\left(\left\{\vec{R}_{l}\right\}\right)=\frac{1}{2} \sum_{i, j=A, B} \sum_{l(i) \neq m(j)} \frac{Z_{i} Z_{j}}{\left|\vec{R}_{l}-\vec{R}_{m}\right|}+E_{g}\left[\rho_{g}(\vec{r}), V_{\text {ext }}\left(\vec{r},\left\{\vec{R}_{l}\right\}\right)\right]
$$

where $\vec{R}_{l}$ are the ionic positions, the sum over $l(i)$ extends over the sites occupied by the $i$-type ions and $\rho_{g}(\vec{r})$ is the ground state electronic density which, according to DFT, is obtained by 
minimizing the energy functional

$$
E[\rho(\vec{r})]=T_{s}[\rho]+E_{\mathrm{ext}}[\rho]+E_{\mathrm{H}}[\rho]+E_{\mathrm{xc}}[\rho],
$$

where the terms represent, respectively, the electronic kinetic energy, $T_{s}[\rho]$, of a non-interacting system with density $\rho(\vec{r})$, the energy of interaction with the external potential due to the ions, $E_{\text {ext }}[\rho]$, the classical electrostatic energy (Hartree term), $E_{\mathrm{H}}[\rho]$ and the exchange-correlation energy, $E_{\mathrm{xc}}[\rho]$, for which we have used the local density approximation. Within the KS-AIMD approach [2] $T_{s}[\rho]$ is calculated exactly by using single particle orbitals whereas the OF-AIMD approach $[1,23]$ uses an explicit albeit approximate expression. Specifically, the functional $T_{s}[\rho]$ includes the von Weizsäcker term plus further terms chosen in order to correctly reproduce some exactly known limits [23].

Another key ingredient is the local ionic pseudopotentials describing the ion-electron interaction. Its construction is fully described in [23] and we just mention that the pseudopotentials have been constructed from first principles by fitting to a model an ion immersed in metallic medium.

Concerning the simulation method, we just briefly mention the main details. We consider $N_{\mathrm{A}}+$ $N_{\mathrm{B}} \equiv N$ ions in a cubic cell with periodic boundary conditions. Given the ionic positions at time $t$, the electronic energy functional is minimized with respect to $\rho(\vec{r})$ represented by a single effective orbital, $\psi(\vec{r})$, defined as $\rho(\vec{r})=\psi(\vec{r})^{2}$. The orbital is expanded in plane waves truncated at a certain cutoff energy. The energy minimization with respect to the Fourier coefficients of the expansion is performed every time step using a quenching method which results in the ground state electronic density and energy. The forces on the ions are obtained from the electronic ground state via the Hellman-Feynman theorem, and the ionic positions and velocities are updated by solving Newton's equations, with the Verlet leapfrog algorithm. In these studies, $N$ ranged from 600 atoms (Li-Mg) to 2000 atoms ( $\mathrm{Li}-\mathrm{Na}$ ), the equilibration time usually lasted between 4 and $5 \mathrm{ps}$ and the calculation of properties was made by averaging over a time interval of 50-90 ps.

\subsection{Collective dynamics}

The density fluctuations in an $\mathrm{A}_{1-x} \mathrm{~B}_{x}$ liquid alloy are usually described by the partial AshcroftLangreth (AL) intermediate scattering functions, $F_{i j}(\vec{q}, t)=\left\langle\rho_{i}(\vec{q}, t) \cdot \rho_{j}^{*}(\vec{q}, 0)\right\rangle$, where $(i, j=A, B)$

$$
\rho_{i}(\vec{q}, t)=\frac{1}{\sqrt{N_{i}}} \sum_{l(i)=1}^{N_{i}} \exp \left[\mathrm{i} \vec{q} \cdot \vec{R}_{l(i)}(t)\right]
$$

is the Fourier transform (FT) of the $i$-type component partial number density, $N_{i}$ is the number of $i$-type particles, $\vec{R}_{l(i)}(t)$ is the position of the $i$-type particle $l$ and $\langle\ldots\rangle$ stands for the ensemble average. The time FT of the $F_{i j}(\vec{q}, t)$ into the frequency domain gives the AL partial dynamic structure factors $S_{\mathrm{ij}}(\vec{q}, \omega)$ which are directly connected with the INS data, namely,

$$
S_{i j}(\vec{q}, \omega)=\frac{1}{2 \pi} \int \mathrm{d} t \mathrm{e}^{\mathrm{i} \omega t}\left\langle\rho_{i}(\vec{q}, t) \cdot \rho_{j}^{*}(\vec{q}, 0)\right\rangle
$$

the asterisk denoting complex conjugation. At some stage, use will be made of the Bhatia-Thornton (BT) concentration-concentration, $S_{\mathrm{CC}}(\vec{q}, \omega)$ and number-number, $S_{\mathrm{NN}}(\vec{q}, \omega)$ partial dynamic structure factors [24], which are linear combinations, weighted by the concentrations, of the AL ones. Another important dynamical magnitude is the $i$-type component particle current

$$
\vec{j}_{i}(\vec{q}, t)=\frac{1}{\sqrt{N_{i}}} \sum_{l(i)=1}^{N_{i}} \vec{u}_{l(i)}(t) \exp \left[\mathrm{i} \vec{q} \cdot \vec{R}_{l(i)}(t)\right]
$$

(with $\vec{u}$ denoting velocity), which is usually split into a longitudinal component, $j_{i}^{\mathrm{L}}(\vec{q}, t)$, parallel to $\vec{q}$, and a transverse component, $j_{i}^{\mathrm{T}}(\vec{q}, t)$, perpendicular to $\vec{q}$. Therefrom, the partial longitudinal, $C_{i j}^{\mathrm{L}}(\vec{q}, t)$, and transverse, $C_{i j}^{\mathrm{T}}(\vec{q}, t)$, current correlation functions are defined

$$
C_{i j}^{\mathrm{L}}(\vec{q}, t)=\left\langle j_{i}^{\mathrm{L}}(\vec{q}, t) \cdot j_{j}^{L *}(\vec{q}, 0)\right\rangle, \quad C_{i j}^{\mathrm{T}}(\vec{q}, t)=(1 / 2)\left\langle j_{i}^{\mathrm{T}}(\vec{q}, t) \cdot j_{j}^{T *}(\vec{q}, 0)\right\rangle
$$


and their time FT's give the respective spectra, $C_{i j}^{\mathrm{L}}(\vec{q}, \omega)$ and $C_{i j}^{\mathrm{T}}(\vec{q}, \omega)$. This study will consider isotropic systems, and therefore all the previous correlation functions depend on $q=|\vec{q}|$ only.

The partial longitudinal current correlation functions are related to the corresponding dynamic structure factors by $C_{i j}^{\mathrm{L}}(q, \omega) \equiv \omega^{2} S_{i j}(q, \omega)$, and therefore they also provide information on the longitudinal collective modes in liquids. Sometimes, it occurs that the diffusive contributions to the partial intermediate scattering functions, $F_{i j}(\vec{q}, t)$, play a dominant role, with the consequence of concealing the oscillations in the associated $S_{i j}(q, \omega)$, which can then be exposed by resorting to the respective $C_{i j}^{\mathrm{L}}(q, \omega)$. For instance, it frequently happens that the $S_{\mathrm{CC}}(q, \omega)$ shows neither side peaks nor shoulders at any $q$-value but they are exposed in the $C_{\mathrm{CC}}^{\mathrm{L}}(q, \omega)$.

The partial transverse current correlation functions, $C_{i j}^{\mathrm{T}}(q, \omega)$, inform about the existence of shear modes in the system. These modes are not connected with any measurable magnitude and can only be analyzed within a theoretical model or by resorting to MD simulations. Among the scant studies on transverse currents in liquids, most have addressed one-component systems. As for binary systems we just mention that CMD simulations have been performed on molten salts, liquid $\mathrm{Li}-\mathrm{Mg}, \mathrm{Li}-\mathrm{Na}$ and $\mathrm{Li}_{4} \mathrm{~Pb}$ alloys and binary Lennard-Jones systems, where optic-like modes have been found $[17,21,25]$.

\section{Results}

\subsection{Li-Na alloy}

The $\mathrm{Li}_{1-x} \mathrm{Na}_{x}$ liquid alloy has been simulated at $T=590 \mathrm{~K}$ and concentrations $x_{\mathrm{Na}}=0.2,0.39$ and 0.60 with respective ionic number densities $0.0368,0.0322$ and 0.0282 (in $\AA^{-3}$ units); further details concerning the input data are given in [26]. The mass ratio for this system is $\approx 3.3$, and it shows strong homocoordinating tendencies.

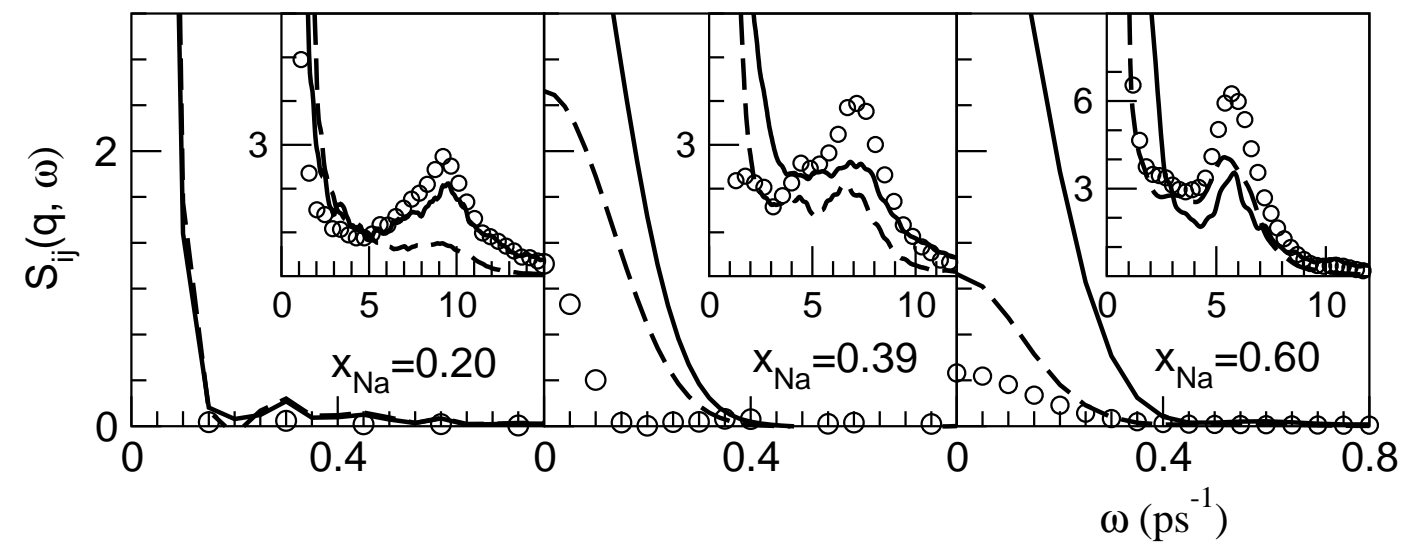

Figure 1. Partial dynamic structure factors, $S_{i j}(q, \omega)$, at $q=0.23 \AA^{-1}$, for the liquid Li-Na alloy at $T=590 \mathrm{~K}$ and three concentrations. Full line: $S_{\mathrm{LiLi}}(q, \omega)$, dashed line: $S_{\mathrm{NaNa}}(q, \omega)$, circles: $S_{\mathrm{NN}}(q, \omega)$. The insets show $10^{3} S_{i j}(q, \omega)$.

Figure 1 depicts, for $q \approx 0.23 \AA^{-1}$, the calculated $S_{\mathrm{LiLi}}(q, \omega), S_{\mathrm{NaNa}}(q, \omega)$ and $S_{\mathrm{NN}}(q, \omega)$ which exhibit clear side peaks at very similar frequencies; this is the expected behaviour in the hydrodynamic regime and represents a propagating acoustic mode. The $S_{\mathrm{NN}}(q, \omega)$ reflects the average behaviour of the system and at the hydrodynamic regime exhibits a clear Rayleigh-Brillouin structure [3]. From the position, $\omega_{\mathrm{B}}$, of the observed Brillouin (side) peaks in the $S_{\mathrm{NN}}(q, \omega)$ at the smallest $q$-value attained by the simulations, $\left(q_{\min } \approx 0.151,0.158\right.$ and $0.165 \AA^{-1}$ for $x_{\mathrm{Na}}=0.60,0.39$ and 0.20 respectively) we can estimate its adiabatic velocity of propagation, $c_{s}=\omega_{\mathrm{B}} / q$. The OF-AIMD calculations give $c_{s}=3100,3000$ and $2530 \mathrm{~m} / \mathrm{s}$ for $x_{\mathrm{Na}}=0.20,0.39$ and 0.60 respectively. There are no experimental data to compare with, but data are available (at $T=590 \mathrm{~K}$ ) for the limits $x_{\mathrm{Na}}=1$ 
(pure $\mathrm{Na}$ ) and $x_{\mathrm{Na}}=0$ (pure Li), where the OF-AIMD calculations give $c_{s}=2330 \pm 80 \mathrm{~m} / \mathrm{s}$ and $c_{s}=4640 \pm 150 \mathrm{~m} / \mathrm{s}$ which are close to the respective experimental values [33] of $\approx 2410$ and $4450 \mathrm{~m} / \mathrm{s}$. Whereas for the three concentrations $S_{\mathrm{LiLi}}(q, \omega)$ shows side peaks up to $q \approx 1.30 \AA^{-1}$, those of $S_{\mathrm{NN}}(q, \omega)$ and $S_{\mathrm{NaNa}}(q, \omega)$ depend on the concentration. The maximum $q$ value for which side peaks appear in $S_{\mathrm{NN}}(q, \omega)$ changes from $q \approx 1.30 \AA^{-1}$ at $x_{\mathrm{Na}}=0.20$ to $q \approx 0.85 \AA^{-1}$ at $x_{\mathrm{Na}}=0.60$; in the case of $S_{\mathrm{NaNa}}(q, \omega)$ the maximum $q$ for which peaks are present ranges from $q \approx 0.40 \AA^{-1}$ at $x_{\mathrm{Na}}=0.20$ to $q \approx 1.1 \AA^{-1}$ at $x_{\mathrm{Na}}=0.60$.

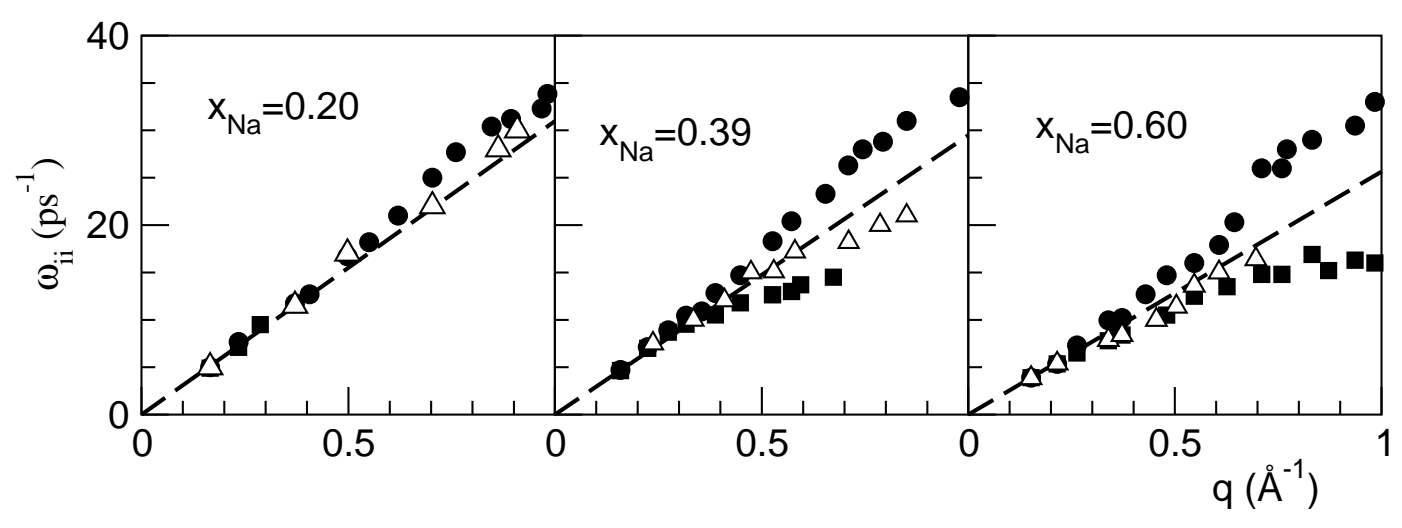

Figure 2. OF-AIMD results for the dispersion curves of the collective modes in $S_{\mathrm{LiLi}}(q, \omega)$, $S_{\mathrm{NaNa}}(q, \omega)$ and $S_{\mathrm{NN}}(q, \omega)$ (full circles, full squares and open triangles respectively) for the liquid $\mathrm{Li}-\mathrm{Na}$ alloy at $T=590 \mathrm{~K}$ and three concentrations. The dashed line stands for the corresponding hydrodynamic adiabatic sound velocities.

From the positions of the side peaks, the associated dispersion curves, $\omega_{\mathrm{LiLi}}(q), \omega_{\mathrm{NaNa}}(q)$ and $\omega_{\mathrm{NN}}(q)$, are obtained (see figure 2 ). Up to $q \approx 0.25 \AA^{-1}, \omega_{\mathrm{LiLi}}(q)$ and $\omega_{\mathrm{NaNa}}(q)$ show for the three concentrations, the same linear behaviour of hydrodynamic collective excitations propagating with the respective adiabatic velocities $c_{s}$. However, above this $q$-value, each dispersion curve splits into two branches exposing two non-hydrodynamic modes, namely the fast and slow sound modes, which signal the onset of a dynamic decoupling between the $\mathrm{Li}$ and $\mathrm{Na}$ particles. The fast mode involves the Li particles only and has a phase velocity, $c_{\text {fast }} \approx 3800 \pm 200 \mathrm{~m} / \mathrm{s}$, which is practically the same for the three concentrations. According to the RET [19,20], the phase velocity of the fast mode should be close to the adiabatic sound velocity of the corresponding light particle fluid. We have asserted it by performing OF-AIMD simulations for pure Li at $590 \mathrm{~K}$ at the same ionic number density as that of the $\mathrm{Li}_{0.61} \mathrm{Na}_{0.39}$ alloy and the adiabatic sound velocity obtained was $3900 \mathrm{~m} / \mathrm{s}$, which is very close to that of the fast mode. Moreover, these results clearly show that as $q$ decreases towards $q_{\mathrm{h}}$, the fast sound mode undergoes a continuous transition into the hydrodynamic sound mode and the process takes place at $0.2 \leqslant q \leqslant 0.4 \AA^{-1}$ which coincides with the range hinted by the CMD calculations [16], and the INS measurements of Bafile et al. [12], for the $\mathrm{He}_{0.77} \mathrm{Ne}_{0.23}$ system. Also, Campa and Cohen [19] have suggested that when the concentration of the light component is increased the fast mode disappears, being overcome by the (extended) sound mode. This can be observed in figure 2 where at $x_{\mathrm{Na}}=0.20$, the dispersion curve $\omega_{\mathrm{LiLi}}(q)$ is rather close to the (extended) hydrodynamic sound mode.

Further information is provided by the longitudinal current correlation functions, $C_{i j}^{\mathrm{L}}(q, \omega)$, which were evaluated according to equation $(6)$. The $C_{\mathrm{LiLi}}^{\mathrm{L}}(q, \omega), C_{\mathrm{NaNa}}^{\mathrm{L}}(q, \omega)$ and $C_{\mathrm{NN}}^{\mathrm{L}}(q, \omega)$ exhibit, for all $q$-values, at least one peak although when the hydrodynamic region is approached both $C_{\mathrm{LiLi}}^{\mathrm{L}}(q, \omega)$ and $C_{\mathrm{NN}}^{\mathrm{L}}(q, \omega)$ exhibit another, low-frequency peak. At the lower $q$-values attained by the simulations, the positions of the peak in $C_{\mathrm{NaNa}}^{\mathrm{L}}(q, \omega)$ as well as the low frequency peaks of $C_{\mathrm{LiLi}}^{\mathrm{L}}(q, \omega)$ and $C_{\mathrm{NN}}^{\mathrm{L}}(q, \omega)$ coincide respectively with the Brillouin peaks in $S_{\mathrm{NaNa}}(q, \omega), S_{\mathrm{LiLi}}(q, \omega)$ and $S_{\mathrm{NN}}(q, \omega)$ which stand virtually at the same position; this is another indication of the hydrodynamic behaviour of the $\mathrm{Li}$ and $\mathrm{Na}$ particles for these small $q$-values. On the other hand, for $x_{\mathrm{Na}}=0.39$ and 0.60 , the high frequency peak of $C_{\mathrm{LiLi}}^{\mathrm{L}}(q, \omega)$ is located at the minimum of the 
$C_{\mathrm{LiNa}}^{\mathrm{L}}(q, \omega)$ which suggests that the $\mathrm{Li}$ and $\mathrm{Na}$ particles oscillate out of phase, i.e. indicates an optic-like mode. From the positions of the previous peaks, the longitudinal dispersion relations, $\omega_{\mathrm{LiLi}}^{\mathrm{L}}(q), \omega_{\mathrm{NaNa}}^{\mathrm{L}}(q)$ and $\omega_{\mathrm{NN}}^{\mathrm{L}}(q)$ are obtained (see figure 3$)$. The $\omega_{\mathrm{NaNa}}^{\mathrm{L}}(q)$ takes smaller values than those of $\omega_{\mathrm{LiLi}}^{\mathrm{L}}(q)$ due to the difference between their respective atomic masses. For all concentrations, the $\omega_{\mathrm{NaNa}}^{\mathrm{L}}(q)$ has one branch whereas the $\omega_{\mathrm{LiLi}}^{\mathrm{L}}(q)$ and $\omega_{\mathrm{NN}}^{\mathrm{L}}(q)$ exhibit for $x_{\mathrm{Na}}=0.39$ and 0.60 two branches, with the low-frequency branch, which has a limited extent, located near the hydrodynamic region. This indicates that in the binary alloy the heavier Na ions keep their characteristic low frequencies whereas the lighter Li ions have a much higher frequency which changes smoothly when approaching the hydrodynamic regime; nevertheless as $q$ decreases some Li ions start catching the low frequency of the heavy Na ions. Obviously, in the hydrodynamic $(q \rightarrow 0)$ limit, all the particles should oscillate with the same frequency and therefore the $C_{\mathrm{LiLi}}^{\mathrm{L}}(q, \omega)$ (and $\left.C_{\mathrm{NN}}^{\mathrm{L}}(q, \omega)\right)$ will only show the low-frequency peak as the high-frequency one vanishes. At low $q$ values, the $\omega_{\mathrm{NaNa}}^{\mathrm{L}}(q)$ has an initial linear increase up to a maximum followed by a minimum located at $q \approx 2.0 \AA^{-1}$ which corresponds to the main peak position of $S_{\mathrm{NaNa}}(q)$. Similar pattern is exhibited by the high-frequency branch of $\omega_{\mathrm{LiLi}}^{\mathrm{L}}(q)$, with a maximum and minimum at $\approx 1.5 \AA^{-1}$ and $2.3 \AA^{-1}$ respectively, which coincide with the first minimum and maximum of $S_{\mathrm{LiLi}}(q)$.

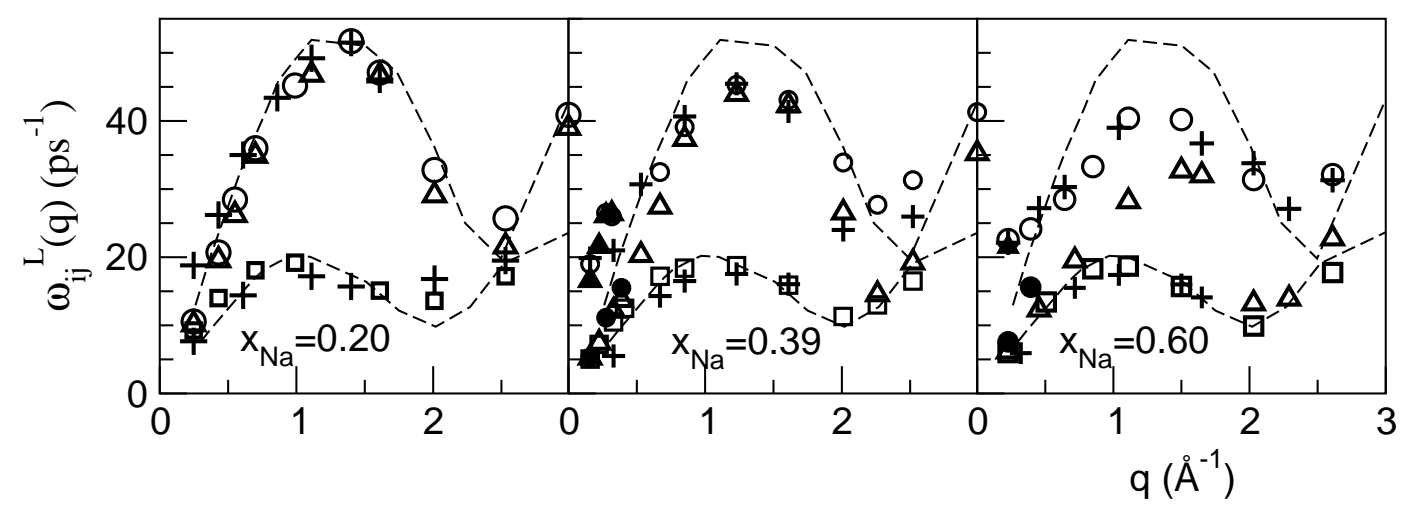

Figure 3. Longitudinal dispersion relation of the partials, $\omega_{\mathrm{LiLi}}^{\mathrm{L}}(q)$ (open and full circles), $\omega_{\mathrm{NaNa}}^{\mathrm{L}}(q)$ (open squares), $\omega_{\mathrm{NN}}^{\mathrm{L}}(q)$ (open and full triangles) and $\omega_{\mathrm{CC}}^{\mathrm{L}}(q)$ (pluses) longitudinal modes for the Li-Na liquid alloy at $T=590 \mathrm{~K}$ and three concentrations. The dashed lines show the longitudinal dispersion relations of pure $\mathrm{Li}$ (upper curve) and $\mathrm{Na}$ (lower curve) at $T=590 \mathrm{~K}$.

At $x_{\mathrm{Na}}=0.39$ and 0.60 , two branches are exhibited by the $\omega_{\mathrm{NN}}^{\mathrm{L}}(q)$ dispersion curve, although the high-frequency branch has a very limited extent, not far from the hydrodynamic region (see figure 3). At these low- $q$ values, the high-frequency branch is induced by the Li particles only, whereas the low-frequency branch comes from both the $\mathrm{Li}$ and $\mathrm{Na}$ particles. As $q$ is increased, the $C_{\mathrm{LiLi}}^{\mathrm{L}}(q, \omega)$ becomes the main contributor to the $C_{\mathrm{NN}}^{\mathrm{L}}(q, \omega)$, and when $\mathrm{Li}$ is the majoritary component, the $\omega_{\mathrm{LiLi}}^{\mathrm{L}}(q)$ and $\omega_{\mathrm{NN}}^{\mathrm{L}}(q)$ are rather close. On the other hand, when Na becomes the majoritary component, the $\omega_{\mathrm{NN}}^{\mathrm{L}}(q)$ stands between both $\omega_{\mathrm{NaNa}}^{\mathrm{L}}(q)$ and $\omega_{\mathrm{LiLi}}^{\mathrm{L}}(q)$. Two branches are also exhibited by the $\omega_{\mathrm{CC}}^{\mathrm{L}}(q)$ dispersion curve which may be connected with propagating concentration modes. The high frequency branch of $\omega_{\mathrm{CC}}^{\mathrm{L}}(q)$ exists for all $q$ values, closely follows the high frequency $\omega_{\mathrm{NN}}^{\mathrm{L}}(q)$ and takes a finite value when $q \rightarrow 0$ which is the typical trend of kinetic modes. The low frequency branch of $\omega_{\mathrm{CC}}^{\mathrm{L}}(q)$ appears just outside the hydrodynamic regime and closely follows $\omega_{\mathrm{NaNa}}^{\mathrm{L}}(q)$. Figure 3 includes the dispersion relations for pure $\mathrm{Li}$ and $\mathrm{Na}$ at $T=590 \mathrm{~K}$. Note that the dispersion relation of the majoritary component in the alloy is very close to that of the pure component whereas that of the minoritary component has a more diffuse structure (less marked maxima and minima) in comparison with the pure component. 


\section{2. $\mathrm{Li}-\mathrm{Mg}$ alloy}

The liquid $\mathrm{Li}_{1-x} \mathrm{Mg}_{x}$ alloy has been studied at three thermodynamic states characterized by $T=887 \mathrm{~K}$, concentrations $x_{\mathrm{Mg}}=0.30,0.50$ and 0.70 and ionic number densities $0.0407,0.0404$ and 0.0401 (in $\AA^{-3}$ units) respectively; further details are provided in [29]. The mass ratio for this system is $\approx 3.4$, and it behaves almost as an ideal mixture in relation to ordering properties.

The calculated $S_{\mathrm{LiLi}}(q, \omega), S_{\mathrm{MgMg}}(q, \omega)$ and $S_{\mathrm{NN}}(q, \omega)$ exhibit clear side peaks which for small $q$ values are located at very similar frequencies; this is the usual behaviour in the hydrodynamic regime, and represents a propagating sound mode. $S_{\mathrm{NN}}(q, \omega)$ exhibits, for $q \leqslant q_{\mathrm{h}}$, a RayleighBrillouin structure, [3] and from the position, $\omega_{\mathrm{B}}$, of its Brillouin peak at the smallest $q$ value reached by the simulations, $\left(q_{\min } \approx 0.23 \AA^{-1}\right)$ we obtain $c_{s}=2400,4000$ and $4550 \mathrm{~m} / \mathrm{s}$ for $x_{\mathrm{Mg}}=$ $0.30,0.50$ and 0.70 respectively. For comparison we note that OF-AIMD calculations for both pure $\mathrm{Li}$ and $\mathrm{Mg}$ near their triple point gave $c_{s}=5000 \pm 150 \mathrm{~m} / \mathrm{s}$ and $c_{s}=4200 \pm 150 \mathrm{~m} / \mathrm{s}$ respectively, whereas its experimental values $[27,28]$ are $\approx 4550$ and $4100 \mathrm{~m} / \mathrm{s}$.

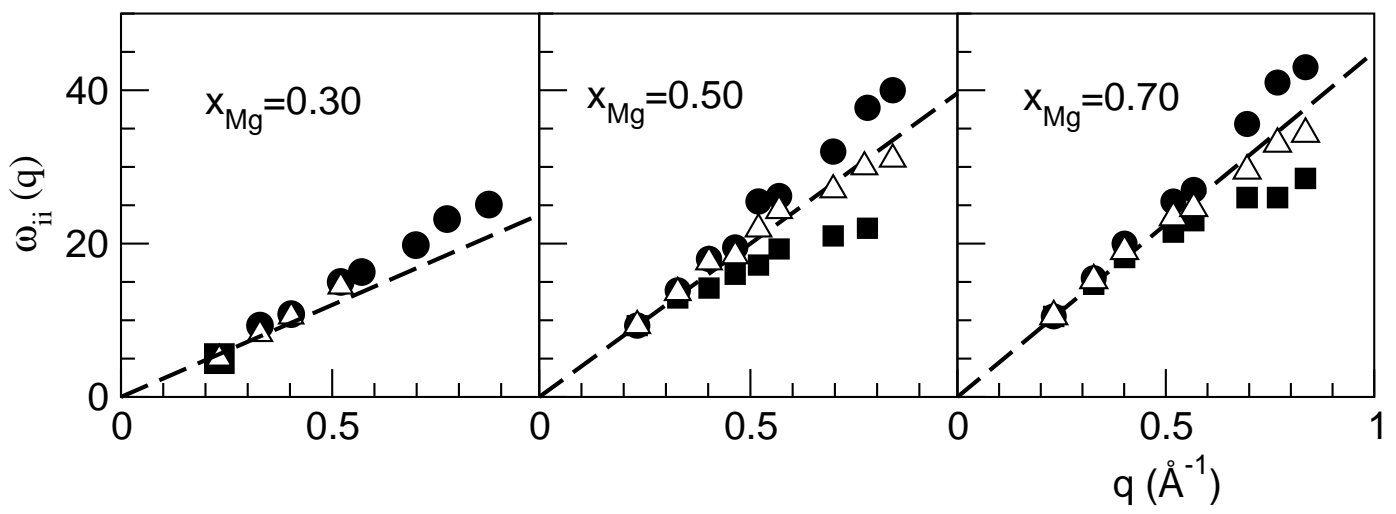

Figure 4. OF-AIMD results for the dispersion curves of the collective modes in $S_{\mathrm{LiLi}}(q, \omega)$ and $S_{\mathrm{MgMg}}(q, \omega)$ and $S_{\mathrm{NN}}(q, \omega)$ (full circles, full squares and open triangles respectively) for the liquid $\mathrm{Li}-\mathrm{Mg}$ alloy at $T=887 \mathrm{~K}$ and three concentrations. The dashed line stands for the corresponding hydrodynamic adiabatic sound velocities.

The range of appearance of the side peaks depends on the concentration, as depicted in figure 4 which shows the associated dispersion curves, $\omega_{\mathrm{LiLi}}(q), \omega_{\mathrm{MgMg}}(q)$ and $\omega_{\mathrm{NN}}(q)$. At $q_{\mathrm{min}}$, the $\omega_{\mathrm{LiLi}}(q), \omega_{\mathrm{MgMg}}(q)$ and $\omega_{\mathrm{NN}}(q)$ virtually coincide, which means that for $q \leqslant q_{\mathrm{min}}$ the $\mathrm{Li}$ and $\mathrm{Mg}$ particles oscillate at the same frequency. But for greater $q$ 's and $x_{\mathrm{Mg}}=0.50$ and 0.70 , the dispersion curve splits into the fast and slow sound modes. The fast mode, involving the Li particles only, has a phase velocity, $c_{\text {fast }} \approx 4600 \pm 200 \mathrm{~m} / \mathrm{s}$, which is virtually the same at both concentrations. Again as $q$ decreases towards $q_{\mathrm{h}}$, the fast sound mode undergoes a continuous transition into the hydrodynamic sound mode and the merging occurs at $0.2 \leqslant q \leqslant 0.3 \AA^{-1}$. Once more, and according with the RET predictions, when the concentration of the light component is increased the fast mode fades away, being overcome by the (extended) sound mode; this is precisely what we observe at $x_{\mathrm{Mg}}=0.30$, where the dispersion curve $\omega_{\mathrm{LiLi}}(q)$ is rather close to the (extended) hydrodynamic sound mode. Additional collective modes can be found by turning to the partial longitudinal currents, $C_{\mathrm{LiLi}}^{\mathrm{L}}(q, \omega), C_{\mathrm{MgMg}}^{\mathrm{L}}(q, \omega), C_{\mathrm{NN}}^{\mathrm{L}}(q, \omega)$ and $C_{\mathrm{CC}}^{\mathrm{L}}(q, \omega)$, from which the corresponding longitudinal dispersion relations $\omega_{\mathrm{LiLi}}^{\mathrm{L}}(q), \omega_{\mathrm{MgMg}}^{\mathrm{L}}(q), \omega_{\mathrm{NN}}^{\mathrm{L}}(q)$ and $\omega_{\mathrm{CC}}^{\mathrm{L}}(q)$ can be derived. However, these dispersion curves are not plotted here since they show no significant departure from the main features already observed for the $\mathrm{Li}-\mathrm{Na}$ alloy, even though the ordering tendencies are very different. We can conclude that the mass ratio is much more important in determining the behaviour of the collective modes than the particular ordering tendencies in the alloy.

We refer now to the transverse collective modes which have been computed for this alloy. As before, from the peak positions of the partial transverse currents, $C_{i j}^{\mathrm{T}}(q, \omega)$, the corresponding transverse dispersion relations, $\omega_{i j}^{\mathrm{T}}(q)$, have been obtained (figure 5$) . \omega_{\mathrm{MgMg}}^{\mathrm{T}}(q)$ has one branch 


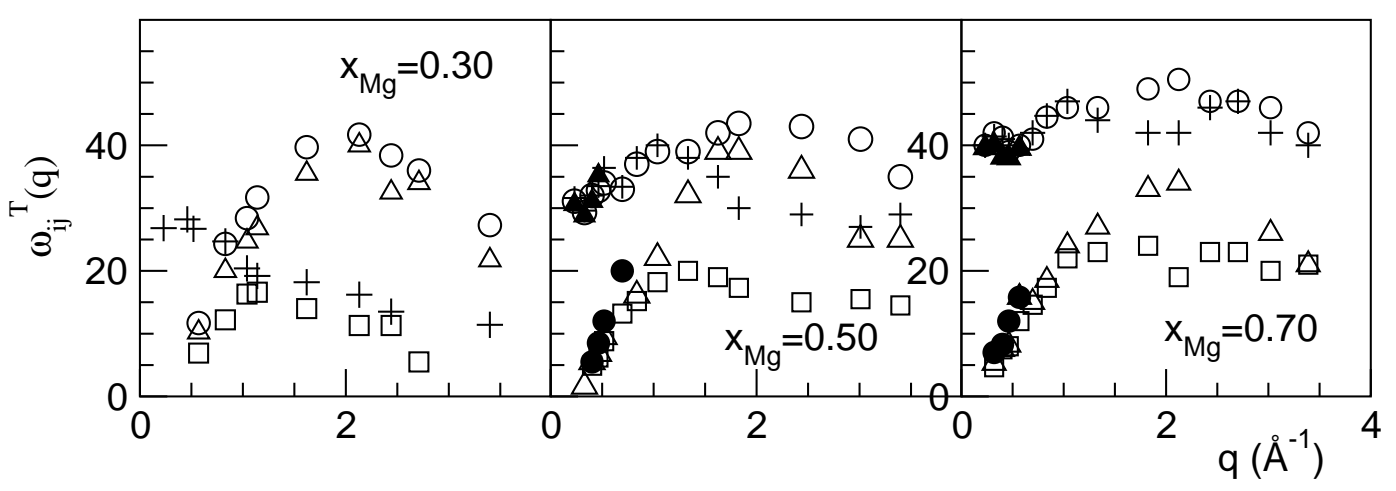

Figure 5. Transverse dispersion relation of the partials, $\omega_{\mathrm{LiLi}}^{\mathrm{T}}(q)$ (open and full circles), $\omega_{\mathrm{MgMg}}^{\mathrm{T}}(q)$ (open squares), number-number, $\omega_{\mathrm{NN}}^{\mathrm{T}}(q)$ (open and full triangles) and concentrationconcentration, $\omega_{\mathrm{CC}}^{\mathrm{T}}(q)$ (pluses) transverse modes for the $\mathrm{Li}-\mathrm{Mg}$ liquid alloy at $T=887 \mathrm{~K}$ and three concentrations.

whereas both $\omega_{\mathrm{LiLi}}^{\mathrm{T}}(q)$ and $\omega_{\mathrm{NN}}^{\mathrm{T}}(q)$ evolve from one to two branches as $x_{\mathrm{Mg}}$ increases; this is the same trend as already observed in the longitudinal dispersion relation. Moreover, the peak in $C_{\mathrm{MgMg}}^{T}(q, \omega)$ and the low frequency peak in the $C_{\mathrm{LiLi}}^{T}(q, \omega)$ coincide with a maximum in the $C_{\mathrm{LiMg}}^{T}(q, \omega)$ which points to in-phase motion of some Li ions with the Mg ions. The low-frequency branch, $\omega_{\mathrm{NN}}^{\mathrm{T}}(q)$ shows typical features of the one-component system, namely it starts at a non-zero value $q_{c}$, exhibits a linear behaviour for low $q$-values, approaching zero as $q \rightarrow q_{c}$. In this linear region the low-frequency branch $\omega_{\mathrm{NN}}^{\mathrm{T}}(q)$ remains close to both $\omega_{\mathrm{MgMg}}^{\mathrm{T}}(q)$ and the low-frequency branch $\omega_{\text {LiLi }}^{\mathrm{T}}(q)$, which implies that the propagation of shear modes involves both species.

The small magnitude of the $C_{\mathrm{CC}}^{T}(q, \omega)$, particularly at small $q$ 's, also underlines a weak contribution to the collective transverse dynamics. However, for the three concentrations we observe clear peaks in the $C_{\mathrm{CC}}^{T}(q, \omega)$ which already exist at $q_{c}$, leading to an $\omega_{\mathrm{CC}}^{\mathrm{T}}(q)$ branch which takes a finite value as $q \rightarrow 0$. The peaks in $C_{\mathrm{CC}}^{T}(q, \omega)$ are a result of both the peak in the $C_{\mathrm{LiLi}}^{T}(q, \omega)$ and a minimum in $C_{\mathrm{LiMg}}^{T}(q, \omega)$ which is related to out-of-phase motion of particles of different species. Similar behaviour in molten salts has been associated with transverse optic modes of kinetic character $[21,25]$.

\subsection{K-Cs alloy}

The longitudinal collective excitations in the liquid $\mathrm{K}_{0.52} \mathrm{Cs}_{0.48}$ alloy have recently been studied using INS experiments [30]. This has prompted us to perform an OF-AIMD study of this system at the same thermodynamic state, namely at $T=300 \mathrm{~K}$ and ionic number density 0.0151 (in $\AA^{-3}$ units) The calculated $S_{\mathrm{KK}}(q, \omega), S_{\mathrm{CsCs}}(q, \omega)$ and $S_{\mathrm{NN}}(q, \omega)$ show clear side peaks within a limited $q$-range. Now $q_{\min }=0.167 \AA^{-1}$, which clearly stands within the hydrodynamic region, and the above partials show side peaks at $\omega_{\mathrm{B}} \approx 3.05 \mathrm{ps}^{-1}$ leading to a value $c_{s} \approx 1820 \pm 150 \mathrm{~m} / \mathrm{s}$.

The associated dispersion curves $\omega_{\mathrm{KK}}(q), \omega_{\mathrm{CsCs}}(q)$ and $\omega_{\mathrm{NN}}(q)$, are plotted in figure 6 . Now, $q_{\mathrm{h}} \approx 0.20 \AA^{-1}$ and for greater $q$-values the dispersion curve splits into the fast and slow sound modes. As $q$ decreases towards $q_{\mathrm{h}}$, the fast sound mode undergoes a continuous transition into the hydrodynamic sound mode at $0.2 \leqslant q \leqslant 0.3 \AA^{-1}$. Note that the mass ratio is now $\approx 3.4$ and the ordering tendencies are almost ideal.

From their INS data Bove et al. [30] derived a dispersion relation for collective excitations by locating the positions of the side peaks in the coherent total dynamic structure factor. Their results are depicted in figure 6 along with the corresponding dispersion relation obtained by the OF-AIMD method, which shows an excellent agreement with experiment. 


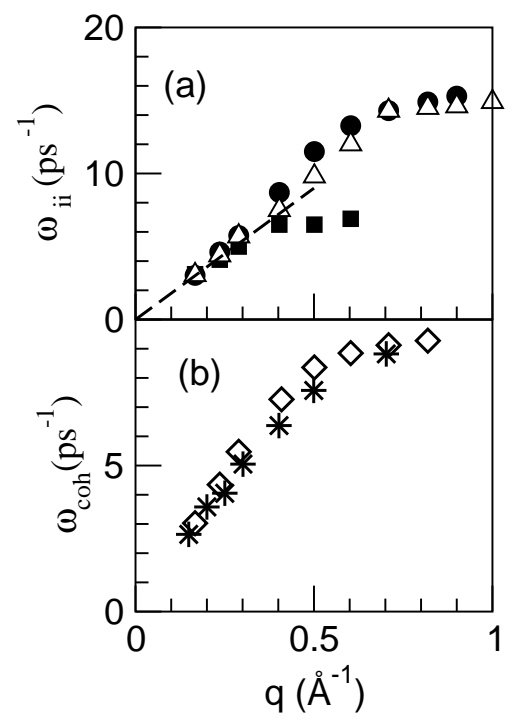

Figure 6. (a) Dispersion curves of the collective modes in $S_{\mathrm{KK}}(q, \omega), S_{\mathrm{CsCs}}(q, \omega)$ and $S_{\mathrm{NN}}(q, \omega)$ (full circles, full squares and open triangles respectively) for the liquid $\mathrm{K}_{0.52} \mathrm{Cs}_{0.48}$ alloy at $T=300 \mathrm{~K}$. The dashed line is the hydrodynamic adiabatic sound velocity. (b) Dispersion relation of the longitudinal collective excitations appearing in the coherent total $S(q, \omega)$. Asterisks: experimental data [30]. Lonzenges: OF-AIMD results.

\subsection{Li-Ba alloy}

OF-AIMD simulations were performed for the liquid $\mathrm{Li}_{1-x} \mathrm{Ba}_{x}$ alloy at $x_{\mathrm{Ba}}=0.12,0.30$ for $T=$ $575 \mathrm{~K}$ and $x_{\mathrm{Ba}}=0.59$ for $T=775 \mathrm{~K}$. These specific thermodynamic states were chosen due to the availability of experimental data for the static structure and several thermodynamic magnitudes. More details are provided in [29]. The mass ratio for this system is the highest considered, $\approx 20$. For $x_{\mathrm{Ba}}=0.12$ and 0.30 the alloy shows weak homocoordinating tendencies, while for Ba concentration 0.59 it has a mild heterocoordinating tendency.

The calculated $S_{\mathrm{LiLi}}(q, \omega), S_{\mathrm{BaBa}}(q, \omega)$ and $S_{\mathrm{NN}}(q, \omega)$ show clear side peaks in a limited $q$ range. Now $q_{\min }=0.19,0.22$ and $0.20 \AA^{-1}$ for $x_{\mathrm{Ba}}=0.12,0.30$ and 0.59 respectively. Interestingly at these $q_{\mathrm{min}}$ the hydrodynamic region has not yet been reached. Whereas at $x_{\mathrm{Ba}}=0.30$ and 0.59 the small difference between the peak positions of the corresponding $S_{i j}(q, \omega)$ suggest that $q_{\min }$ should be close to $q_{\mathrm{h}}$, however for $x_{\mathrm{Ba}}=0.12$ there is still an appreciable mismatch between the peak positions of $S_{\mathrm{LiLi}}(q, \omega)$ and $S_{\mathrm{BaBa}}(q, \omega)$ (located at $\approx 5.1$ and $3.7 \mathrm{ps}^{-1}$ respectively). Therefore, the transition towards the hydrodynamic regime takes place at $q$ values which are clearly smaller than those found in all previous alloys. Thereby the hydrodynamic regime comprises a smaller range of wavevectors, as the increased mass difference makes it harder for the light Li ions to oscillate with the same frequency as the much heavier Ba ions due to the increased difference between their oscillation frequencies. From the position of the side peaks in $S_{\mathrm{NN}}(q, \omega)$, we have estimated the adiabatic sound velocity in the alloy, i.e. $c_{s}=2500,2000$ and $1700 \mathrm{~m} / \mathrm{s}$ for $x_{\mathrm{Ba}}=0.12,0.30$ and 0.59 which compare well with the experimental [32] values of 2550,1900 and $1500 \mathrm{~m} / \mathrm{s}$ respectively.

Figure 7 shows the dispersion relations derived from the peak positions. For the three concentrations there appears a splitting into fast and slow modes and the difference in slopes between both modes is substantially greater than in the previous alloys. This is a consequence of the greater mass difference (and therefore, the oscillation frequencies) between the Li and Ba ions. Notice that the fast sound mode still exists at the smallest concentration of the heavy Ba particles, namely $x_{\mathrm{Ba}}=0.12$; these findings confirm the RET predictions that the increase of the mass ratio and/or the decrease of the number density widens the concentration range for the existence of both modes.

From the calculated partial longitudinal current correlation functions and the positions of their respective peaks we have obtained the corresponding longitudinal dispersion relations which are plotted in figure 8. These dispersion relations show the same basic trends already found for the 


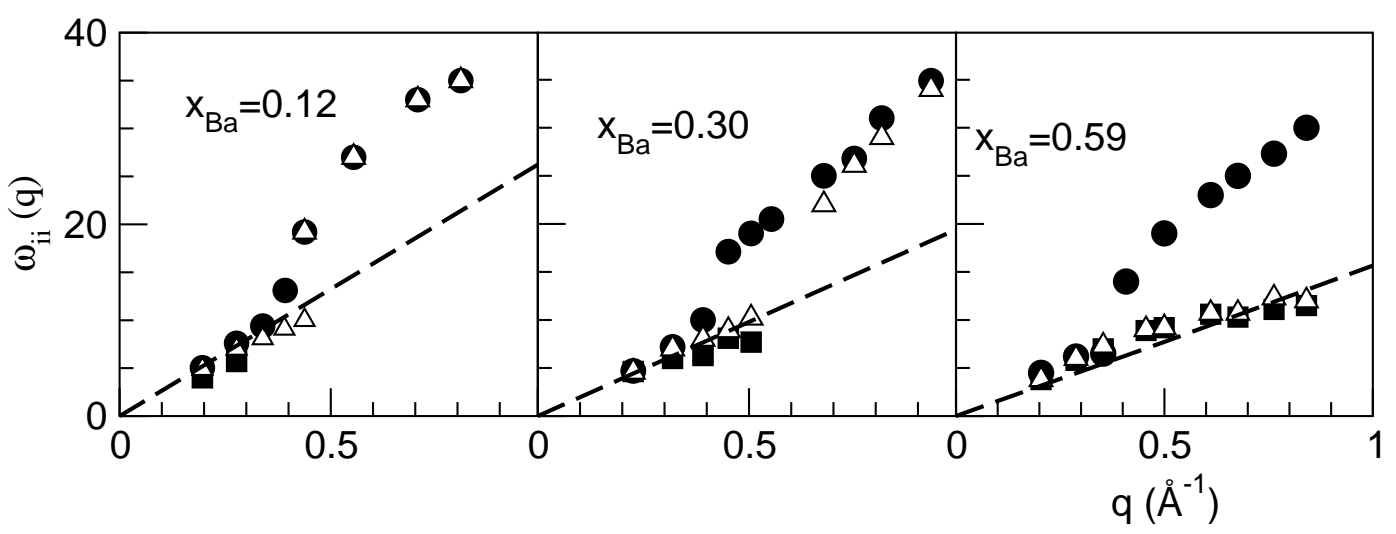

Figure 7. OF-AIMD results for the dispersion curves of the collective modes in $S_{\mathrm{LiLi}}(q, \omega)$ and $S_{\mathrm{BaBa}}(q, \omega)$ and $S_{\mathrm{NN}}(q, \omega)$ (full circles, full squares and open triangles respectively) for the liquid $\mathrm{Li}-\mathrm{Ba}$ alloy at the three concentrations considered in this work. The dashed line stands for the corresponding hydrodynamic adiabatic sound velocities.

other liquid alloys, namely (i) the heavier component has one dispersion branch, $\omega_{\mathrm{BaBa}}^{\mathrm{L}}(q)$, which hardly changes with concentration, (ii) both the lighter component, $\omega_{\mathrm{LiLi}}^{\mathrm{L}}(q)$, and $\omega_{\mathrm{NN}}^{\mathrm{L}}(q)$ evolve from one to two branches as the concentration of the heavier component increases, and (iii) the $\omega_{\mathrm{CC}}^{\mathrm{L}}(q)$ always has a high-frequency branch which takes a finite value when $q \rightarrow 0$, as well as a low-frequency one which disappears when the concentration of the heavier component increases.

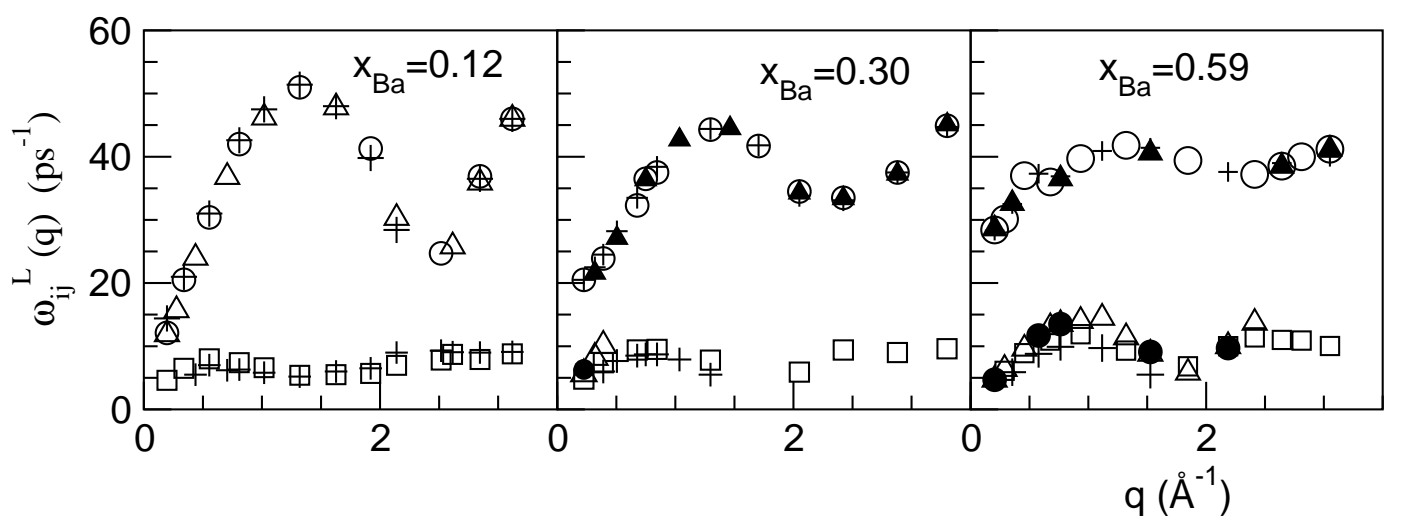

Figure 8. Longitudinal dispersion relation of the partials, $\omega_{\mathrm{LiLi}}^{\mathrm{L}}(q)$ (open and full circles), $\omega_{\mathrm{BaBa}}^{\mathrm{L}}(q)$ (open squares), number-number, $\omega_{\mathrm{NN}}^{\mathrm{L}}(q)$ (open and full triangles) and concentrationconcentration, $\omega_{\mathrm{CC}}^{\mathrm{L}}(q)$ (pluses) longitudinal modes for the Li-Ba liquid alloy at the three concentrations considered in this work.

The transverse currents, $C_{i j}^{\mathrm{T}}(q, \omega)$, have been calculated and the associated transverse dispersion relations, $\omega_{i j}^{\mathrm{T}}(q)$, are plotted in figure $9 . \omega_{\mathrm{BaBa}}^{\mathrm{T}}(q)$ always has one branch whereas both $\omega_{\mathrm{LiLi}}^{\mathrm{T}}(q)$ and $\omega_{\mathrm{NN}}^{\mathrm{T}}(q)$ develop a second branch as the concentration of the heavier component increases. However, at $x_{\mathrm{Ba}}=0.12$, the $\omega_{\mathrm{LiLi}}^{\mathrm{T}}(q)$ and $\omega_{\mathrm{NN}}^{\mathrm{T}}(q)$ have only one branch which virtually coincides with the high-frequency $\omega_{\mathrm{CC}}^{\mathrm{T}}(q)$ and they take a finite value as $q \rightarrow 0$, which signals the absence of propagating shear modes. The $\omega_{\mathrm{CC}}^{\mathrm{T}}(q)$ always has one branch connected with the minoritary component whereas at $x_{\mathrm{Ba}}=0.12,0.30$ a second branch appears. Moreover, the low frequency, $\omega_{\mathrm{CC}}^{\mathrm{T}}(q)$ branch starts well outside the linear region and this suppression of the CC modes in the long wavelength limit is predicted by the GCM model [21] for those systems with a high mutual diffusion and a tendency towards homocoordination. 


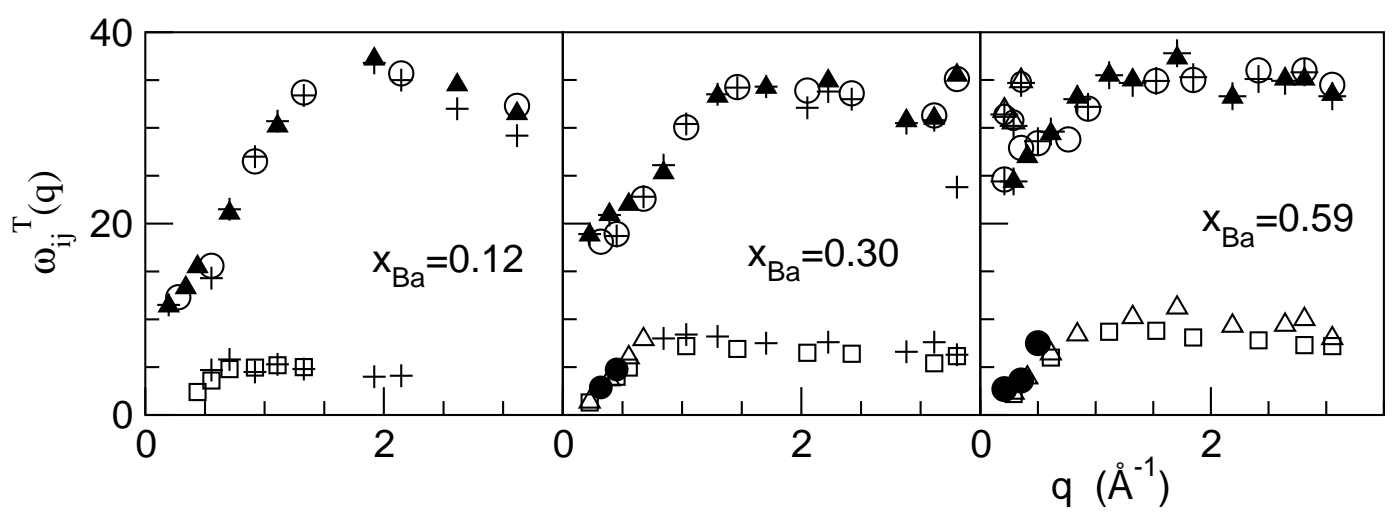

Figure 9. Transverse dispersion relation of the partials, $\omega_{\text {LiLi }}^{\mathrm{T}}(q)$ (open and full circles), $\omega_{\mathrm{BaBa}}^{\mathrm{T}}(q)$ (open squares), number-number, $\omega_{\mathrm{NN}}^{\mathrm{T}}(q)$ (open and full triangles) and concentrationconcentration, $\omega_{\mathrm{CC}}^{\mathrm{T}}(q)$ (pluses) transverse modes for the Li-Ba liquid alloy.

\section{Conclusions}

We have shown that $a b$ initio MD simulations can be used in order to study the collective dynamical properties of liquid binary alloys. The advantage of using orbital free methods lies in the ability to study large simulation systems so that the hydrodynamic region of wavevectors can be reached from above to study the evolution of the collective modes in this region. This method has been applied for the specific case of several $s-p$ bonded liquid alloys for which the previous studies on several static structural properties showed a good agreement with experimental data. Now, several collective longitudinal and transversal properties have been evaluated for various compositions. Although the scant experimental data available for the dynamical properties do not allow a thorough comparison theory/experiment, we do find good agreement where experiments are available ( $\mathrm{K}-\mathrm{Cs}$ dispersion relation, and $\mathrm{Li}-\mathrm{Ba}$ adiabatic sound velocity). Nonetheless, it must be stressed that similar OF-AIMD calculations performed for the pure components (for which a wider range of experimental data is available) showed a good agreement with experiment.

For all alloys, the calculated partial dynamic structure factors, $S_{\mathrm{AA}}(q, \omega)$ and $S_{\mathrm{BB}}(q, \omega)$ ( $\mathrm{A}=$ light element and $\mathrm{B}=$ heavy element), show clear side peaks which extend far beyond the hydrodynamic regime and represent two non-hydrodynamic modes known as the fast and slow sound modes. This phenomenon had already been predicted by the RET when applied to a binary mixture of hard spheres [19], but it had also been concluded that a mass ratio larger than 10 was required in order to expose the non-hydrodynamic modes. The present ab initio calculations show that those modes may also appear in systems with a significant smaller mass ratio, $\approx 3$. Investigation of alloys with even smaller mass ratios, like $\mathrm{Na}-\mathrm{K}$ or Ga-In (where it is $\approx 1.7$ ), would be of interest in order to discern the possible disappearance of this type of modes. Furthermore, for all the systems studied we have found that as the wavevector $q$ is decreased towards the hydrodynamic region, the fast and slow sound modes smoothly merge into the hydrodynamic sound mode. We should stress that, in contrast with some rare gas mixtures [20] or hard-spheres mixtures [19], we have never found an abrupt disappearance of one of the modes while the other goes into the hydrodynamic one, but in all cases a smooth merging has been observed, and this is so irrespective of the mass ratio and of the ordering tendencies. This process takes place at a range of $q$ values which becomes smaller as the mass ratio increases. In this way, whereas for the $\mathrm{Li}-\mathrm{Na}$ and $\mathrm{Li}-\mathrm{Mg}$ alloys it occurs at around $0.2 \leqslant q \leqslant 0.4 \AA^{-1}$, for the Li-Ba alloy the range moves towards smaller values, which in the $\mathrm{Li}_{0.88} \mathrm{Ba}_{0.12}$ alloy happens at $q \leqslant 0.2 \AA^{-1}$.

Another remarkable feature is the appearance of two branches for $\omega_{\mathrm{NN}}^{\mathrm{L}, \mathrm{T}}(q), \omega_{\mathrm{AA}}^{\mathrm{L}, \mathrm{T}}(q)$ and $\omega_{\mathrm{CC}}^{\mathrm{L}, \mathrm{T}}(q)$, in both the longitudinal and transverse dispersion relations, with the high-frequency branches representing kinetic modes which are overdamped. However, there is only one branch for $\omega_{\mathrm{BB}}^{\mathrm{L}}(q)$ which just weakly depends on the concentration. 
The low-frequency $\omega_{\mathrm{NN}}^{\mathrm{L}}(q)$ and $\omega_{\mathrm{AA}}^{\mathrm{L}}(q)$, along with the $\omega_{\mathrm{BB}}^{\mathrm{L}}(q)$, go linearly to zero at $q=0$ and they represent acoustic modes. On the other hand, the low-frequency $\omega_{\mathrm{NN}}^{\mathrm{T}}(q)$ and $\omega_{\mathrm{AA}}^{\mathrm{T}}(q)$, along with the $\omega_{\mathrm{HH}}^{\mathrm{T}}(q)$, go linearly to zero at a finite $q$-value, $q_{c}$, and they represent propagating shear modes. However, the low-frequency $\omega_{\mathrm{CC}}^{\mathrm{T}}(q)$ branch does not exist for low $q$-values as it appears just outside the linear region; this behaviour is consistent with the predictions of the GCM model. Moreover, we find that by increasing the concentration of the lighter component, the range of the low-frequency $\omega_{\mathrm{NN}}^{\mathrm{T}}(q)$ branch is diminished.

Summing up, our results show that the basic difference among the (longitudinal and transverse) dispersion relations of the liquid alloys considered in this work, is just a quantitative one, stemming from the greater atomic mass difference between the alloy components. For instance, it shows up in a wider gap between the $\omega_{\mathrm{BaBa}}^{\mathrm{L}, \mathrm{T}}(q)$ and the high-frequency $\omega_{\mathrm{LiLi}}^{\mathrm{L}, \mathrm{T}}(q)$ curves, a shrinkage of the hydrodynamic regime, i. e., a decrease of $q_{\mathrm{h}}$, as well as in the fact that the collective motions (acoustic and shear waves) are progressively hindered with increasing atomic mass mismatch.

\section{Acknowledgements}

We acknowledge the financial support of the DGICYT of Spain (MAT2005-03415), the EU FEDER program and JCyl (VA068A06). 


\section{References}

1. Hohenberg P., Kohn W., Phys. Rev., 1964, 136, B864.

2. Kohn W., Sham L.J., Phys. Rev., 1965 140, A1133.

3. Balucani U., Zoppi M. Dynamics of the Liquid State. Oxford. Clarendon, 1994; Hansen J.P., McDonald I.R. Theory of Simple Liquids. Academic, London, 1986.

4. Sjögren L., Sjölander A., J. Phys. C, 1979 12, 4369; Sjögren L., J. Phys. C, 1979, 13, 705; Sjögren L., Phys. Rev. A, 1980, 22, 2866; Sjögren L., Phys. Rev. A, 1980, 22, 2883.

5. Casas J., González D.J., González L.E., Alemany M.M.G., Gallego L.J., Phys. Rev. B, 2000 $62,12095$.

6. Jacucci G., McDonald I.R., J. Phys. F: Metal Phys. 1980, 10 L15.

7. Jacucci G., Ronchetti M., Schirmacher W., J. Physique Coll., 19848 C8, 385.

8. Crevecoeur R.M., Smorenburg H.E., de Schepper I.M., J. Low Temp Phys., 1996, 105149.

9. Montfrooij W., Westerhuijs P., de Haan V.O., de Schepper I.M., Phys. Rev. Lett., 1989, 63, 544.

10. de Jong P.H.K., Verkerk P., de Vroege C.F., de Graaf L.A., Howells W.S., Bennington S.M., J. Phys.: Condens. Matter, 1994, 6 L681.

11. Alvarez M., Bermejo F.J., Verkerk P., Roessli B., Phys. Rev. Lett., 1998, 80, 2141.

12. Bafile U., Verkerk P., Guarini E., Barocchi F., Phys. Rev. Lett., 2001, 86, 1019.

13. Schram R.P.C., Bot A., Schaink H.M., Wegdam G.H., J. Phys.: Condens. Matter, 1991, 2 SA157; Schram R.P.C., Wegdam G.H. Bot A., Phys. Rev. A, 1991, 44, 8062.

14. Enciso E., Almarza N.G., Dominguez P., Bermejo F.J., Phys. Rev. Lett., 1995, 74, 4233.

15. Fernandez-Perea R., Alvarez M., Bermejo F.J., Verkerk P., Roessli B., Enciso E., Phys. Rev. E, 1998, $\mathbf{5 8}, 4568$.

16. Sampoli M., Bafile U., Guarini E., Barocchi F., Phys. Rev. Lett., 2002, 88, 085502.

17. Anento N., Padro J.A., Mol. Phys., 2001, 99, 275.

18. Bosse J., Jacucci G., Ronchetti M., Schirmacher W., Phys. Rev. Lett., 1986, 57, 3277.

19. Campa A., Cohen E.G.D., Phys. Rev. A, 1990, 41, 5451.

20. Westerhuijs P., Montfrooij W., de Graaf L.A., de Schepper I.M., Phys. Rev. A, 1992, 45, 3749.

21. Bryk T., Mryglod I., Kahl G., Phys. Rev. E, 1997, 56, 2903.; Bryk T., Mryglod I., Phys. Lett. A, 1999, 261, 349.; J. Phys.: Condens. Matter, 2000, 12, 6063; Bryk T., Mryglod I., Condens. Matter Phys., 2004, 7, 285; Bryk T., Mryglod I., J. Phys.: Condens. Matter, 2005, 17, 413.

22. Blanco J., González D.J., González L.E., López J.M., Stott M.J., Phys. Rev. B, 2003, 67, 041204.; González D.J., González L.E., López J.M., Stott M.J., Eurohys. Lett., 2003, 62, 42.; Phys. Rev. E, 2004, 69, 031205.

23. González D.J., González L.E., López J.M., Stott M.J., Phys. Rev. B, 2002, 65, 184201.

24. Bhatia A.B., Thornton D.E., Phys. Rev. B, 1970, 2, 3004.

25. Adams E.M., McDonald I.R., Singer K., 1977, Proc. R. Soc. A, 357, 37.

26. González D.J., González L.E., López J.M., Stott J.M., Phys. Rev. E, 2004, 69, 031205.

27. Hornung K. Handbook of Thermodynamic and Transport Properties of Alkali Metals. Blackwell, Oxford, 1985.

28. Iida T., Guthrie R.I.L. The Physical Properties of Liquid Metals. Clarendon Press, Oxford 1988.

29. González D.J., González L.E., López J.M., Stott M.J., J. Phys.: Condens. Matter, 2005, 171429.

30. Bove L.E., Sacchetti F., Petrillo C., Dorner B., Phys. Rev. Lett., 2000, 85, 5352.

31. Kim M.G., Letcher S.V., J. Chem. Phys. 1971, 55, 1164.

32. Saar J., Ruppersberg H., Phys. Chem. Liq., 1987, 1745.

33. Beyer R.T. Handbook of Thermodynamic and Transport Properties of Alkali Metals. Blackwell, Oxford, 1985 . 


\title{
Мікроскопічна динаміка у рідких бінарних сплавах: дослідження методом безорбітальної першопринципної молекулярної динаміки
}

\author{
Д.Х.ґонзалез, Л.Е.ґонзалез \\ Відділ теоретичної фізики, Університет Вальядоліда, Вальядолід, Іспанія \\ Отримано 30 жовтня 2007 р.
}

\begin{abstract}
Ми повідомляємо дослідження методом першопринципної молекулярної динаміки колективних збуджень у декількох рідких бінарних сплавах з $s$-р-зв'язком. Результати подано для $\mathrm{Li}-\mathrm{Na}$, Li-Mg, K-Cs та Li-Ba рідких сплавів з різними концентраціями, для яких співвідношення мас $€$ в області від $\approx 3$ для Li-Na до $\approx 20$ для Li-Ba, та змінними тенденціями впорядкування, від сильної гомокоординації у $\mathrm{Li}-\mathrm{Na}$ до помірної гетерокоординації для одної концентрації Li-Ba. Дослідження було проведено методом безорбітальної першопринципної молекулярної динаміки у комбінації з локальними іонними псевдопотенціалами, побудованими в рамках цього ж підходу. Ми аналізуємо залежність колективних мод від концентрації та співвідношення мас компонент сплаву, та знаходимо спільну поведінку для всіх систем незважаючи на різні тенденції впорядкування.
\end{abstract}

Ключові слова: першопринципне моделювання, бінарні сплави, колективна динаміка, повздовжні колективні збудження, поперечні колективні збудження

PACS: $61.25 . \mathrm{Mv}, 61.55 . \mathrm{Hg}, 61.20 . \mathrm{Ja}$ 